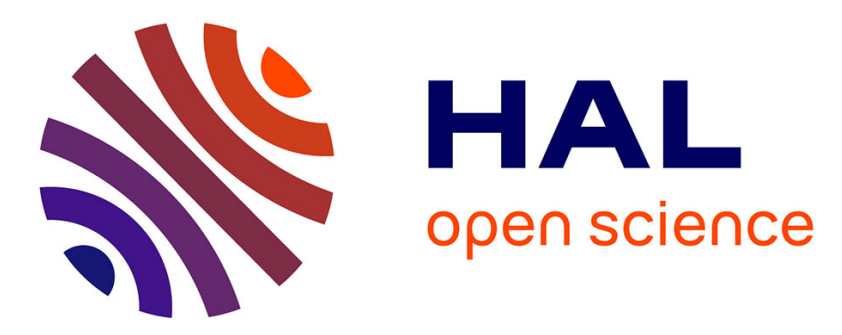

\title{
Shadow electrochemiluminescence microscopy of single mitochondria
}

Yumeng Ma, Camille Colin, Julie Descamps, Stéphane Arbault, Neso Sojic

\section{To cite this version:}

Yumeng Ma, Camille Colin, Julie Descamps, Stéphane Arbault, Neso Sojic. Shadow electrochemiluminescence microscopy of single mitochondria. Angewandte Chemie International Edition, 2021, 60 (34), pp.18742-18749. 10.1002/anie.202105867 . hal-03424312

\section{HAL Id: hal-03424312 \\ https://hal.science/hal-03424312}

Submitted on 10 Nov 2021

HAL is a multi-disciplinary open access archive for the deposit and dissemination of scientific research documents, whether they are published or not. The documents may come from teaching and research institutions in France or abroad, or from public or private research centers.
L'archive ouverte pluridisciplinaire HAL, est destinée au dépôt et à la diffusion de documents scientifiques de niveau recherche, publiés ou non, émanant des établissements d'enseignement et de recherche français ou étrangers, des laboratoires publics ou privés. 


\title{
Shadow electrochemiluminescence microscopy of single mitochondria
}

\author{
Yumeng Ma, Camille Colin, Julie Descamps, Stéphane Arbault, ${ }^{*, \dagger}$ and Neso Sojic* \\ Y. Ma, Dr. C. Colin, J. Descamps, Dr. S. Arbault, Prof. N. Sojic \\ University of Bordeaux, Bordeaux INP, ISM, UMR CNRS 5255, 33607 Pessac, France \\ E-mails: stephane.arbault@u-bordeaux.fr; neso.sojic@enscbp.fr \\ †Present address: Univ. Bordeaux, CNRS, Bordeaux INP, CBMN UMR 5248, Allée Geoffroy Saint Hilaire, 33600 Pessac, France
}

\begin{abstract}
Mitochondria are the subcellular bioenergetic organelles. The analysis of their morphology and topology is essential to provide useful information on their activity and metabolism. Herein, we report a labelfree shadow electrochemiluminescence (ECL) microscopy based on the spatial confinement of the ECLemitting reactive layer to image single living mitochondria deposited on the electrode surface. The ECL mechanism of the freely-diffusing $\left[R u(b p y)_{3}\right]^{2+}$ dye with the sacrificial tri-n-propylamine coreactant restrains the light-emitting region to a micrometric thickness allowing to visualize individual mitochondria with a remarkable sharp negative optical contrast. The imaging approach named "shadow ECL" (SECL) reflects the negative imprint of the local diffusional hindrance of the ECL reagents by each mitochondrion. The statistical analysis of the colocalization of the shadow ECL spots with the functional mitochondria revealed by classical fluorescent biomarkers, MitoTracker Deep Red and the endogenous intramitochondrial NADH, validates the reported methodology. The versatility and extreme sensitivity of the approach are further demonstrated by visualizing single mitochondria, which remain hardly detectable with the usual biomarkers. Finally, by alleviating problems of photobleaching and phototoxicity associated with conventional microscopy methods, SECL microscopy should find promising applications in the imaging of subcellular structures.
\end{abstract}

\section{Introduction}

Analytical studies of the mitochondria status in bioenergetics and physio-pathological processes are usually performed based on bulk and large population measurements. ${ }^{[1]}$ Both cells and isolated mitochondria have been studied using spectrophotometric, UV-visible absorption or fluorescence sensors, and electrochemical methods, including the oxygen consumption sensing by the so-called Clark electrode. ${ }^{[2]}$ Fluorescence spectroscopy and imaging led to major scientific outputs such as the monitoring of membrane potential variations, NADH (reduced form of nicotinamide adenine dinucleotide) and FAD (flavine adenine dinucleotide) changes along the oxidative phosphorylation states as well as the characterization of Reactive Oxygen Species production by mitochondria ${ }^{[3]}$ In the search of structure-function relationships for physio-pathological studies, a strong development of mitochondrial imaging has occurred over the last two decades. The goals were first to image the mitochondrial network evolution, its disorganization in correlation with genetic defects or carcinogenic processes, the mitochondria shape and ultrastructure in the network, etc. ${ }^{[4]}$ Recent developments of complex molecular sensors allow to measure an increasing number of biological markers and transitory regulators of mitochondrial bioenergetics, including calcium ions, phosphate-based substrates, hypochlorite ions, sulfur dioxide, hydrogen peroxide, etc. ${ }^{[5]}$ All these progresses as well as improvements in imaging techniques lead to the monitoring of single active mitochondria. ${ }^{[6]}$ These approaches allow first to assess the span of individual activities within mitochondrial population, the existence of sub-populations and to gain resolution on dynamic events at single mitochondria. Striking examples are reported for the observation of membrane potential oscillations or transitory drops due to the activity of the mitochondrial Permeability Transition Pore (mPTP) under calcium supply. ${ }^{[7]}$ Such events participate to physio-pathological dysfunctions, for instance in cardiac injuries or cancers. ${ }^{[8]}$ Consequently, a clear need exists for the development of bioimaging methods with a single mitochondrion resolution. ${ }^{[9]}$

Electrochemiluminescence $(E C L)$ is the light emission triggered by an electrochemical process. ${ }^{[10]}$ The initial electron-transfer reactions occurring at the electrode surface provoke a cascade of homogeneous reactions involving highly reactive intermediates and lead in fine to the generation of the excited state of a dye. ${ }^{[11]}$ Its radiative relaxation provides an optical readout, which enables the development of various microscopy approaches. ${ }^{[12]}$ Thanks to its extremely low background, high sensitivity, absence of photobleaching effects and autofluorescence, and precise electrochemical control of the reactivity in space and time, ECL microscopy is a fast developing research area. ${ }^{[12-13]}$ The field explores very diverse applications, ranging from the visualization of the latent fingerprints, redox interfacial processes, single entities such as nanowires, attoliter droplets, molecular wires, micro/nanoparticles used in electrocatalytic processes and for immunosensing. ${ }^{[14]}$ 
Recently, great attention has been focused on the imaging of single cells. ${ }^{[12-13,14 k, 15]}$ Hydrogen peroxide, glucose and intracellular sphingomyelinase activity have been measured using the luminol emitter. ${ }^{[16]}$ An important breakthrough in the development of ECL imaging was the demonstration of the surface-confined ECL microscopy of cellular membranes and proteins labeled with a $\left[R u(b p y){ }_{3}\right]^{2+}$-derivative dye operating in heterogeneous format. ${ }^{[17]}$ An in situ coreactant-embedded ECL method based on tertiary amine conjugated polymer dots was also described to detect membrane proteins. ${ }^{[18]}$ In this context, label-free imaging is an appealing alternative and several groups have achieved the ECL visualization of single cells. ${ }^{[19]}$ Particularly relevant to our work are findings recently reported by Su et al. ${ }^{[19 a, 20]}$ They imaged by ECL the cell-matrix adhesions located on a silica nanochannel membrane and cell-cell junctions. Zhang et al. reported the morphological characterization of cells captured on an electrode surface by ECL imaging. ${ }^{[19 b]}$ However, ECL microscopy remains limited at the level of single cells and imaging subcellular organelles was not reported so far. $^{[12-13,17-19,21]}$

Thus, we report herein the ECL microscopy of specific subcellular organelles by imaging single living mitochondria using a label-free approach (Scheme 1). Mitochondrial membrane composition and permeation properties are different from cell plasma membranes. Dyes targeting mitochondria comprise positively-charged fluorescent dyes such as rhodamine derivatives (e.g. tetramethylrhodamine methyl ester, rhodamine 123) or MitoTracker dyes. The first ones are sequestered in the matrix space of polarized mitochondria due to the strong potential difference across the internal membrane, but they are also released once mitochondria experience a loss of polarization (oxidative phosphorylation activity), whereas the second category reacts chemically with the protein thiol groups in the mitochondrial matrix giving a permanent labelling even for nonactive mitochondria. However, these labels aimed at targeting mitochondria can interfere with the mitochondrial metabolism and respiration. ${ }^{[22]}$ Consequently, label-free methods might be particularly well-adapted for imaging active mitochondria. ${ }^{[23]}$

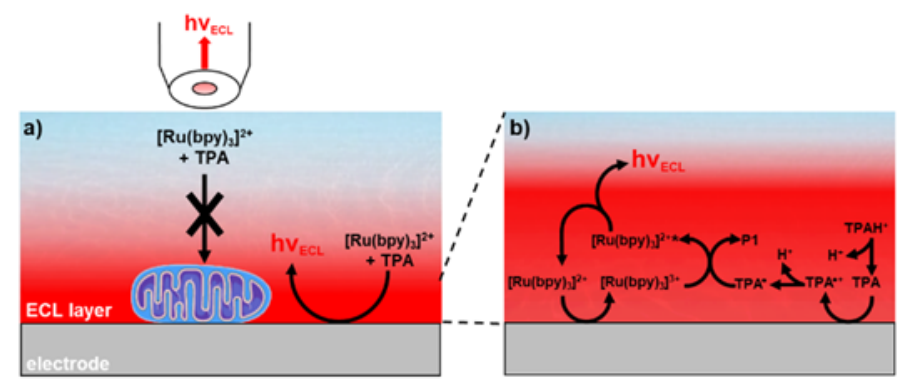

Scheme 1. a) Side-view scheme of the label-free shadow ECL microscopy (SECL) of single mitochondria based on the spatial confinement of the ECL-emitting layer. b) The ECL mechanism involving the direct oxidation of both $\left[\mathrm{Ru}(\mathrm{bpy})_{3}\right]^{2+}$ and TPA ("direct oxidation" route) restrains the spatial extension of the lightemitting layer (vide infra).

In this work, we describe a straightforward methodology based on the remarkable negative optical contrast provided by shadow ECL (SECL) to image in a label-free manner single functional mitochondria isolated from Saccharomyces cerevisiae yeasts. The model ECL system consisting of the freely-diffusing $\left.[R u(b p y)]_{3}\right]^{2+}$ dye and of the very efficient sacrificial tri-n-propylamine (TPA) coreactant was used in a homogeneous format to visualize individual mitochondria sedimented on the electrode surface. Diffusional hindrance of the ECL reagents by the single mitochondria leads to a lower local light emission. The ECL layer was spatially confined to the immediate vicinity of the electrode surface due to the ECL mechanism (Scheme $1 \mathrm{~b}$ ) and the thickness of the ECL-emitting layer is thus comparable with the size of the investigated biological entity, i.e. the mitochondrion. The ECL negative contrast imaging of mitochondria colocalizes with their staining by a specific dye, MitoTracker Deep Red, ${ }^{[24]}$ and with the endogenous mitochondrial NADH (autofluorescence molecule). The performances and the versatility of the presented surface-confined SECL microscopy open new perspectives to image subcellular structures and non-labeled objects.

\section{Results and Discussion}

The concept of SECL microscopy is presented on Scheme 1 for imaging single mitochondria deposited on a glassy carbon (GC) electrode surface. To minimize the interferences with the mitochondrial metabolism or membrane potential, our goal was to avoid any surface functionalization or mitochondria pretreatment, which was reported for covalent binding or irreversible adsorption. ${ }^{[6,25]}$ Indeed, mitochondria are specific organelles with membrane structures different from cell plasma membranes. They do not present external glycocalyx matrix but a double membrane comprising a high protein content and specific phospholipids. In the inner membrane are embedded the redox complexes of the respiratory chain, aimed at generating a membrane 
potential used by the ATP synthase to produce the pro-energetic ATP molecules. In this work, a suspension of isolated mitochondria was let to sediment on the GC electrode surface. The hydrophilic interactions between the surface and the mitochondria let them remain immobile for at least $30 \mathrm{~min}$. permitting their sharp visualization by ECL and fluorescence. ECL emission was generated at the electrode surface by imposing a potential of $1.45 \mathrm{~V} \mathrm{vs}$. Ag/AgCl/KCl 3M (all the potentials in this work are reported vs. this reference electrode) to the GC electrode immersed in a PBS solution containing the freely-diffusing $E C L$ reagents (i.e. $[R u(b p y)]^{2+}$ and TPA). This anodic potential was high enough to oxidize both reagents and generate ECL emission. Figure 1a shows a typical ECL image recorded by a CCD camera (exposure time of $200 \mathrm{~ms}$ ) of single mitochondria deposited on the GC electrode (Figure S1). Once sedimented on the GC surface, single mitochondria are easily observed and well-resolved spatially (Figure 1).
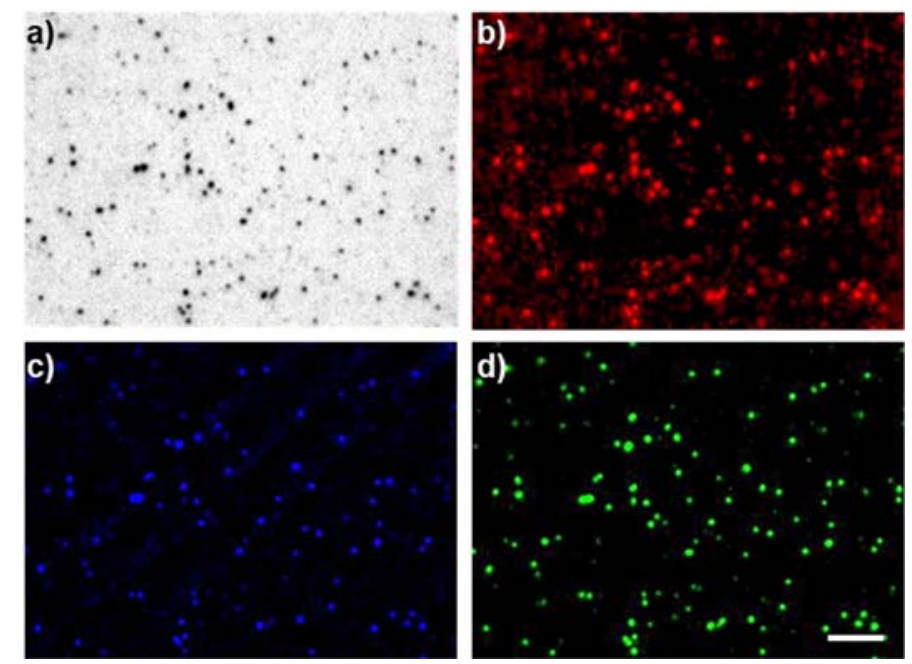

Figure 1. Images of single mitochondria deposited on a GC electrode obtained by (a) SECL, (b) SECL with an inverted color-coded representation (see text for details), (c) fluorescence detection of endogenous NADH and of (d) MitoTracker Deep Red dye. The same ROI was imaged with the different microscopies. SECL was recorded in PBS solution ( $\mathrm{pH}=7.4$ ) containing $30 \mu \mathrm{M}\left[\mathrm{Ru}(\mathrm{bpy})_{3}\right]^{2+}$ and $100 \mathrm{mM}$ TPrA by applying $1.45 \mathrm{~V}$. Scale bar: $10 \mu \mathrm{m}$.

The entire electrode surface generated ECL light except at the level of dark spots, which corresponded to single mitochondria (Figure 1a). Indeed, the deposited mitochondria hindered locally the diffusion of the ECL reagents to the electrode surface and thus inhibited the initial electrochemical reactions, which lead to the ECL emission. The fact that mitochondria appeared darker than the bare GC surface showed that the ECL reagents cannot diffuse through the mitochondria as reported for cells. ${ }^{[17,19 a, 19 b]}$ On one hand, this diffusional hindering behavior is not a surprising result considering the fact that these entities are surrounded by both inner and outer membranes. On the other hand, due to the respiratory chain reactions and the resulting transmembrane electrochemical potential, the internal mitochondrial membrane is negatively charged whereas the ECL dye is positively charged and accumulation of $\left[\mathrm{Ru}(\mathrm{bpy})_{3}\right]^{2+}$ may happen. In the solution containing the ECL reagents, we recorded the photoluminescence $(\mathrm{PL})$ images of the electrode surface where mitochondria were sedimented, by using the dichroic filters adapted to the $\left[R u(b p y){ }_{3}\right]^{2+} \mathrm{PL}$, and we have not been able to observe the mitochondria in these conditions. It indicates that this accumulation effect is negligible. Moreover, even if the ECL dye may concentrate at the level of the mitochondrial membrane, it does not generate noticeable ECL emission at $1.45 \mathrm{~V}$ as demonstrated by the dark spots (Figure 1a). To investigate further this point and the ECL mechanism, we recorded first the voltammetric and ECL signals on GC with the same ECL solution (Figure S2). The less anodic "ECL wave" is observed at potentials where only TPA is oxidized at the electrode surface (typically $0.8 \mathrm{~V}$ ) and it follows the mechanistic pathway called "low oxidation potential" route (Scheme $\mathrm{S} 1)^{\left[{ }^{[26]}\right.}$ The second more anodic "ECL wave" appears at potential above $\sim 0.9 \mathrm{~V}$ where $\left[\mathrm{Ru}(\mathrm{bpy})_{3}\right]^{2+}$ is significantly oxidized (Figure S2). When a potential of $0.8 \mathrm{~V}$ was imposed, we did not observe any ECL emission (Figure S3) unlike the case of $\left[\mathrm{Ru}(\mathrm{bpy})_{3}\right]^{2+}$-decorated beads. ${ }^{[14 j, 27]}$ It demonstrates clearly that the "low oxidation potential" ECL mechanism does not contribute notably at the level of the mitochondria in comparison to the mechanism(s) operating on the bare GC electrode surface (vide infra) where ECL is much stronger, even if $\left[\mathrm{Ru}(\mathrm{bpy})_{3}\right]^{2+}$ could accumulate at the mitochondrial membrane. In fact, the presented SECL strategy enabled visualization of the projected mitochondria bodies on the electrode surface as dark spots resembling shadows in the surrounding ECL However, it is important to emphasize that the shadow pattern is not a projection of their structures on the electrode surface due to the ECL light emitted above them. Dark spots observed in SECL microscopy reflects only on the local hindrance of the diffusional flux of the ECL reagents by the mitochondria. Conceptually, this diffusional shadow presents some similarities with the 
negative feedback mode of Scanning Electrochemical Microscopy performed on insulating objects such as cells. ${ }^{[28]}$ To facilitate the visual perception of structure and contrast of mitochondria in the ECL images, and the comparison with fluorescence images obtained with NADH and MitoTracker Deep Red (Figure 1c-d), the grey scale of the SECL images was inverted so that the bare electrode surface appeared dark (i.e. corresponding to strong ECL intensity) and the mitochondria bright, coded in red color (i.e. corresponding to low ECL intensity) as illustrated on Figure 1b.

Before recording the SECL images, the same region of interest (ROI) was imaged according to the fluorescence of endogenous intramitochondrial NADH content (Figure 1c) and an exogenous dye, MitoTracker Deep Red (Figure 1d). We confronted our SECL images to the fluorescence of these two model dyes because (1) they are classically used to image and study mitochondria; (ii) they are specific to mitochondria; (iii) they are complementary since the first exogenous dye reflects the existence of the membrane potential and the second endogenous one the functional metabolism of the mitochondria; (iv) the fluorescence images of these two dyes may be recorded even in the presence of the dissolved $\left[R u(b p y)_{3}\right]^{2+}$. On one hand, MitoTracker Deep Red is a commercially available fluorescent probe that labels mitochondria utilizing the mitochondrial membrane potential to accumulate in the matrix. On the other hand, $\mathrm{NADH}$ is the redox substrate of the internal NADH dehydrogenases, which transfer protons and electrons to the respiratory chain and fuel the oxidative phosphorylation and, as such, informs on the respiratory chain status. In other words, fluorescence signals of MitoTracker Deep Red and of NADH are the signatures of the effectiveness of the mitochondrial integrity and activity. The exposure times of the CCD to acquire well-resolved images were $5 \mathrm{~s}, 5 \mathrm{~s}$ and $0.2 \mathrm{~s}$ for NADH, MitoTracker Deep Red, and SECL, respectively. This difference in the exposure time (i.e. longer for fluorescence than for SECL) showed also the remarkable sensitivity and optical contrast achieved by the SECL approach.

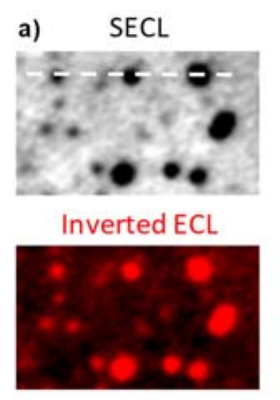

NADH

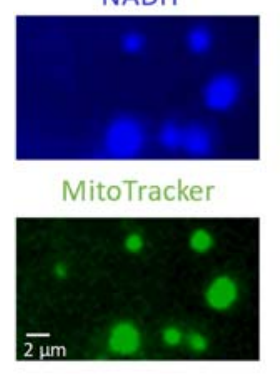

b)
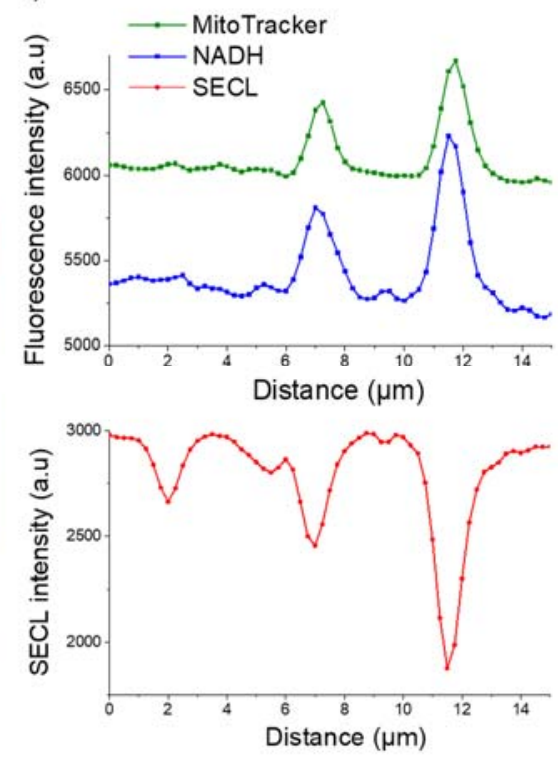

c)

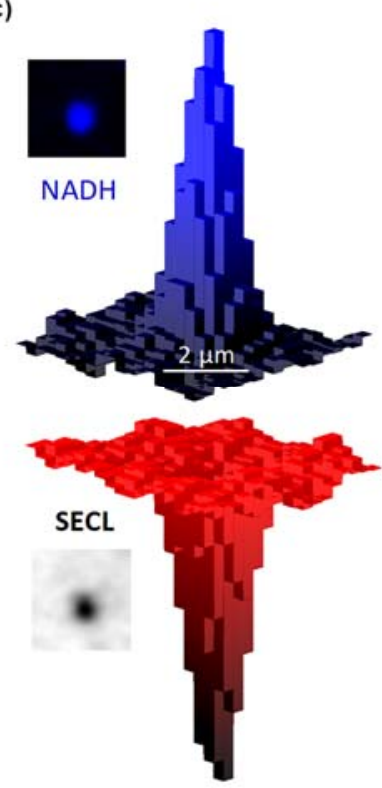

Figure 2. a) Typical SECL and fluorescence images recorded at high magnification of the same ROI showing single mitochondria. b) Luminescence intensity profiles extracted along the dashed line materialized on the top image in (a). c) An example of 3-D imaging of a single mitochondrion recorded by NADH fluorescence (top) and SECL (bottom). Same experimental conditions as in Figure 1.

As already mentioned, the shadow-ECL spots reveal the location of the mitochondria on the electrode surface. On the ROI shown in Figure 1a, one can observe 118 single mitochondria by SECL. On Figure 1c, the NADH fluorescent spots showed the structural integrity of the mitochondria as well as the metabolic activity. The spatial distribution of NADH fluorescence (Figure 1c) follows a pattern very similar to the SECL image (Figure 1b). A total of $\sim 85$ mitochondria are observable in Figure $1 \mathrm{c}$ based on NADH autofluorescence. Fluorescence images recorded with MitoTracker Deep Red are very similar to both NADH and SECL images. It confirms that the shadow ECL spots correspond to the mitochondria present on the electrode surface. In addition, fluorescence from MitoTracker Deep Red proves that the mitochondria are polarized in these experimental conditions (Figure 1d). In addition, since similar NADH and MitoTracker Deep Red fluorescence levels have been obtained on the mitochondria before and after SECL imaging (Figure S4), it indicates that the ECL experimental conditions did not alter their metabolic redox status. On Figure $1 \mathrm{~b}$, a number of 118 mitochondria were observed by inverted ECL whereas 85 and 115 by NADH (Figure 1c) and MitoTracker Deep Red (Figure 1d) fluorescence, respectively. These differences in detectable mitochondria number between SECL and 
fluorescence detections can be explained by the remarkable sensitivity of SECL due to an improved optical contrast.

Then, we compared image quality of SECL with NADH and MitoTracker fluorescence microscopy. Figure 2 shows a typical example of SECL and fluorescence images of the same ROI with a higher magnification. 7 mitochondria were revealed by MitoTracker Deep Red fluorescence. Only 6 were visible according to the NADH autofluorescence. They corresponded mainly to the larger mitochondria. In SECL, these 7 entities were easily visible but 5 smaller mitochondria were also resolved. Consistently, luminescence intensity profile could be extracted along the dashed line on Figure 2a. The NADH and MitoTracker fluorescence profiles shows two peaks whereas SECL profile reveals an additional peak at $2.5 \mu \mathrm{m}$ and a shoulder at $6 \mu \mathrm{m}$ position. These last two were visible only by using the SECL approach. While structural details were limited by the conventional optical diffraction limit, the SECL microscopy produced sharp and detail-rich images of the single mitochondria (Figure 2c). Figure 2c exemplifies at the level of a mitochondrion in 3-D the good agreement between the SECL decrease with the NADH fluorescence variations. This comparative study illustrates the remarkable sensitivity of SECL to image small mitochondria, which were hardly detectable by NADH and MitoTracker fluorescence.
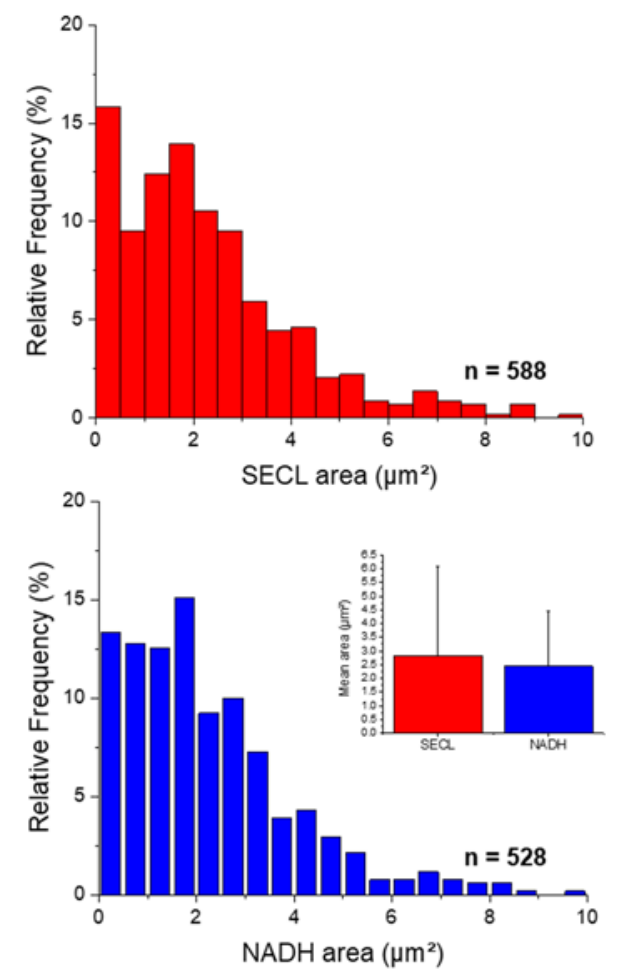

Figure 3. Statistical distributions of the mitochondria size (luminescent spot area) measured from (a) SECL and (b) NADH autofluorescence images. The inset displays the mean area value of the mitochondria measured by SECL and NADH autofluorescence over 588 and 528 single mitochondria, respectively.

To investigate further the imaging abilities offered by SECL for subcellular entities, we confronted the SECL images to the NADH fluorescence ones. We selected the NADH autofluorescence signal because living mitochondria are hallmarked by the intramitochondrial presence of $\mathrm{NADH}$, estimated in the sub-millimolar concentration range. ${ }^{[29]}$ Figure S5a shows the overlay of both luminescence signals for the same ROI. One can observe a clear similarity for most of the mitochondria. However, as already mentioned, the smallest mitochondria are imaged only by SECL. We then analyzed the luminescence intensity of each pixel from both SECL and NADH fluorescence images (Figure S5b). A statistical analysis of the cross-correlation between both detection modes resolved at the single pixel level shows a general trend in which pixels with a higher $\mathrm{NADH}$ fluorescence intensity correlate with a lower SECL intensity. Our data reveal a strong positive correlation (Pearson's coefficient $r=0.72$ ) between both intensities. Since high NADH fluorescence signal corresponds usually to larger mitochondria, the strong correlation indicates that the larger the mitochondria are, the lower the local SECL intensity measured.

The comparison of SECL and NADH fluorescence images allowed subsequent statistical analysis of the distributions of the mitochondria sizes based on the measurement of their luminescent spot area. 588 single functional mitochondria were analyzed comparatively by SECL and fluorescence on the same ROls (4 experiments). The resulting histogram measured on SECL images is displayed on Figure $3 a$. The size distribution showed that the projected area of the majority of the mitochondria was smaller than $2 \mu m^{2}$. The 
averaged area values of the mitochondria, were $\sim 2.5 \mu \mathrm{m}^{2}$ for SECL and NADH fluorescence (inset in Figure 3 ). These values are in good agreement with the average size of isolated mitochondria, typically ranging from $0.4 \mu \mathrm{m}$ to $1.8 \mu \mathrm{m}$ in length corresponding to surfaces between about 0.5 to $6 \mu \mathrm{m}^{2}$. ${ }^{[25,30]}$ Only $13 \%$ of mitochondria with an area $\leq 0.5 \mu \mathrm{m}^{2}$ were observable by NADH fluorescence whereas it represents $16 \%$ of objects detected by SECL. This small difference between SECL and NADH fluorescence imaging was also reflected in the total number of mitochondria detected by SECL and NADH fluorescence (588 and 528, respectively) over the same ROI. However, we cannot completely exclude that some of the small SECL spots correspond to non-functional mitochondria. Whatever, it confirmed that smaller mitochondria were better detected by SECL, as mentioned previously. On the other hand, the mitochondria might appear smaller in SECL than in fluorescence of NADH because the borders of the mitochondria may not hinder completely the diffusion of the ECL reagents and thus they do not block completely the local ECL generation. Nevertheless, analysis of the size distribution demonstrated clearly that smaller mitochondria were revealed and observed by SECL in comparison to fluorescence.

SECL microscopy is based on the diffusional hindrance of the ECL reactants by the mitochondrion, which can be considered as an entity blocking the direct electron-transfer reactions from the electrode surface to the ECL reagents. Therefore, we investigated the extent of diffusion hindrance as a function of the mitochondrion size. The ECL intensity measured at the level of each mitochondrial spot was plotted as a function of its projected area. Figure 4 illustrates the dependence of the mean SECL intensity with the area of the corresponding mitochondrion. The statistical analysis of 171 entities shows a clear trend between the decrease of the ECL intensity with the mitochondrial size (projected area). The ECL intensity measured locally at the bare GC surface (i.e. electrode surface without mitochondria) was 3000 a.u. For the smallest mitochondria (area $<1$ $\mu \mathrm{m}^{2}$ ), the ECL signal diminished to ca. 2700 a.u., representing a decrease of $10 \%$ versus the pristine value. With larger mitochondria, the ECL intensity continued to decrease monotonically. With mitochondria covering typically $8 \mu \mathrm{m}^{2}$ (possibly several aggregated entities), the ECL fell to 2200 a.u., so approximately a decrease of ca. $30 \%$. In other words, the larger the mitochondria are, the higher hindrance of the diffusional flux of both ECL reagents to the electrode surface is achieved and the lower local ECL signal is measured. This observation is supported by SECL experiments on larger biological entities (mammalian Chinese Hamster Ovary cells with typical dimensions of $30 \mu \mathrm{m}$ ) with a drop of ECL intensity of ca. $90 \%$ (unpublished results). This is in agreement with recent reports on other cell lines. ${ }^{[19 a, 20]}$

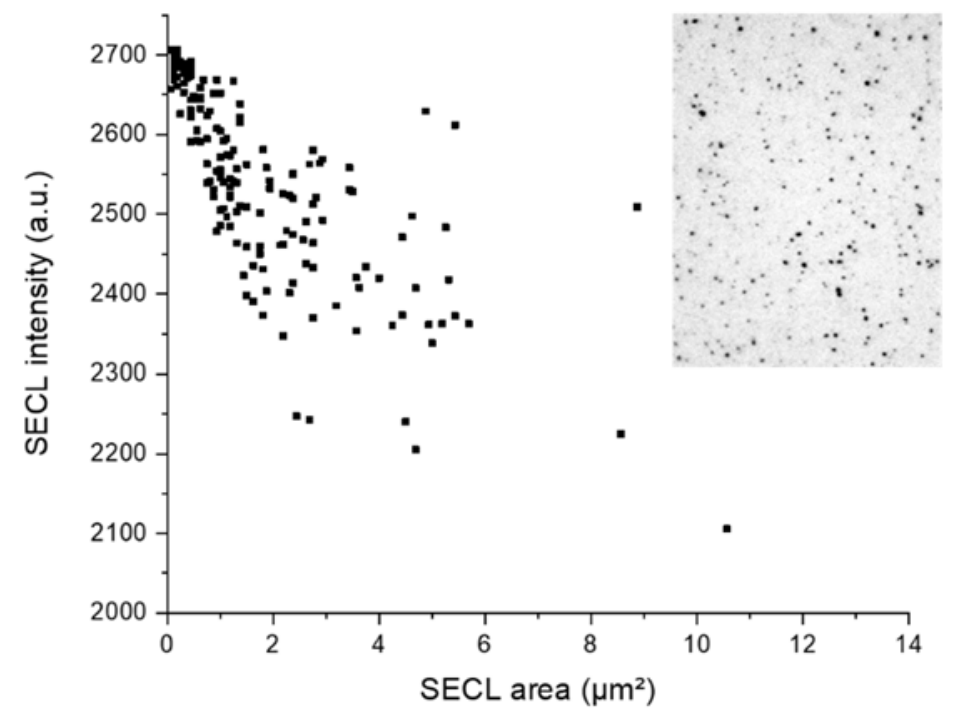

Figure 4. Study of the correlations between SECL intensity and projected area of the mitochondria measured individually ( $n=171$ ). The inset shows the SECL image used to extract the values of the SECL intensity and the corresponding area for each mitochondrion.

In the present case, the fact that ECL was not completely suppressed at the level of the mitochondrial spots can be explained by two main factors. The first one is related to the diffusional hindrance of the ECL reagents. The oblong shape of the mitochondria may not block completely the electrode surface resulting in a partial ECL signal. In addition, the possible distance between the electrode surface and the outer mitochondria membrane may allow partial diffusion of the ECL reagents and local generation of low ECL signal. For instance, it is known that the plasma membrane of cells is separated from the substrate by $100 \mathrm{~nm}-150 \mathrm{~nm}$ via the formation of extracellular matrices-integrin-cytoskeleton linkages. ${ }^{[31]}$ Even if mitochondria present a completely different structures from mammalian cells, we cannot exclude the existence of such a small interstitial space between the outer mitochondrial membrane and the GC surface that cannot be resolved with classic microscopy techniques. Nevertheless, all the mitochondria are visualized in the same focal plane at 
the electrode surface and they remain immobile enough for at least $30 \mathrm{~min}$. owing to weak interactions with the GC surface. It indicates that this eventual interstitial distance is extremely small, even if it might affect the SECL signal. In fact, in future works, the analysis of the SECL signal could be a possible method to estimate this distance with the support of simulations. The second possibility is related to the thickness of the ECLemitting layer in comparison to the typical dimensions of a mitochondrion as well as to the distribution of the ECL intensity in this reactive layer. The SECL experiments have been performed with $30 \mu \mathrm{M}[\mathrm{Ru}(\mathrm{bpy}) 3]^{2+}$ in presence of TPA in excess (i.e. $100 \mathrm{mM}$ ). Several competitive mechanistic pathways may occur simultaneously and allow ECL generation at some distance from the electrode surface. ${ }^{[26,32]}$ The thickness of the $E C L$ reaction layer depends on the electrode material, on the respective concentrations of $\left[\mathrm{Ru}(\mathrm{bpy})_{3}\right]^{2+}$ and TPA, on the $\mathrm{pH}$, the imposed potential, the hydrophilicity of the electrode surface, etc. ${ }^{[33]}$ This ECL system involving $\left[\mathrm{Ru}(\mathrm{bpy})_{3}\right]^{2+}$ and TPA is still an active research area with extensive mechanistic and characterization studies. [141, 26-27, 32a, 34]

We can consider three predominant ECL routes: the "low oxidation potential" route (as already mentioned, $\mathrm{ECL}$ emission occurs before the oxidation of $\left[\mathrm{Ru}(\mathrm{bpy})_{3}\right]^{2+}$; Scheme S1), the "catalytic" route (it follows an EC' mechanism; see Scheme S2), and the "direct oxidation" route (both ECL reagents are directly oxidized at the electrode surface; Schemes $1 \mathrm{~b}$ and 2). Considering the respective concentrations of $\left[R u(b p y){ }_{3}\right]^{2+}$ and TPA, the homogeneous catalytic oxidation of TPA by electrogenerated $\left[R u(b p y)_{3}\right]^{3+}$ occurs slowly at micromolar concentrations (herein, $30 \mu \mathrm{M}$ ), implying that the contribution of the "catalytic" route is negligible. Indeed, Bard and coworkers reported that this route plays a predominant role for $\left[\mathrm{Ru}(\mathrm{bpy})_{3}\right]^{2+}$ concentrations $>\sim 0.5 \mathrm{mM},{ }^{[35]}$ well above the concentration used in the present work. In addition, the "direct oxidation" route became more important at more anodic potentials. ${ }^{[35]}$ Therefore, since we have not observed any ECL emission at $0.8 \mathrm{~V}$ (i.e. the "low oxidation potential" route), we can conclude that the "direct oxidation" route prevails in our experimental conditions. This ECL route can be described by the following reaction scheme:[32a, 35-36]

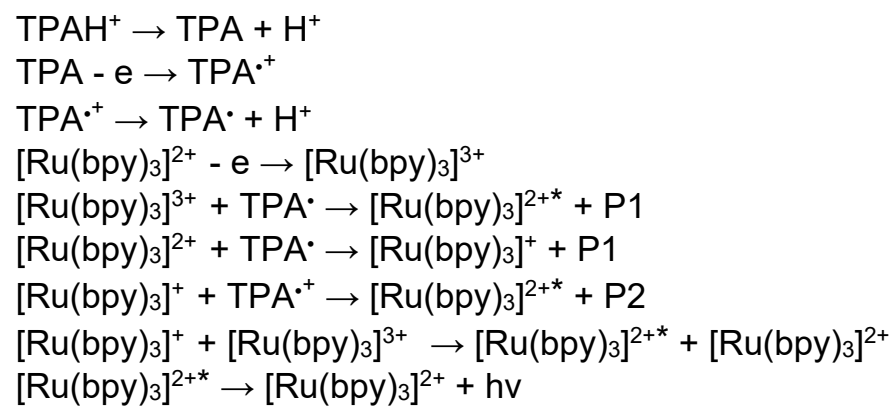

Scheme 2. Sequence of reactions involved in the "direct oxidation" route. P1 and P2 are the iminium cation or similar byproducts. ${ }^{[26,32 a]}$

The oxidation of both $\left[\mathrm{Ru}(\mathrm{bpy})_{3}\right]^{2+}$ and TPA occurs concomitantly at the electrode surface. The electrogenerated TPA ${ }^{*+}$ radical deprotonated and form a strongly reducing radical, TPA ${ }^{*}$. The electron-transfer reaction between TPA ${ }^{\cdot}$ and $\left[R u(b p y)_{3}\right]^{3+}$ is very exergonic and populates the excited state, which leads to the $\mathrm{ECL}$ emission. In brief, the ECL process confines mechanistically the generation of the excited state of the $\left[\mathrm{Ru}(\mathrm{bpy})_{3}\right]^{2+}$ dye very close to the electrode surface. Recently, Su and coworkers imaged nicely the thickness of ECL layer at gold microtube electrodes. ${ }^{[37]}$ In presence of $25 \mathrm{mM}$ TPA, ECL intensity was the highest at the electrode surface and then decreased very rapidly with estimated experimental and simulated values of the entire ECL layer between ca. 1.5 and $4 \mu \mathrm{m}$, depending on the $\left[\mathrm{Ru}(\mathrm{bpy})_{3}\right]^{2+}$ concentration. However, the majority of the ECL signal originated from a distance $<1 \mu \mathrm{m}$ from the electrode surface. ECL self-interference spectroscopy was also used by the same group to estimate the thickness of the ECL reactive layer and they obtained values between $\sim 350 \mathrm{~nm}$ and $1 \mu \mathrm{m}$, depending on the experimental conditions. ${ }^{[38]}$ The central point is the confinement of the ECL-emitting layer to the electrode surface. In addition, as already mentioned, considering the different mechanistic pathways, the most important contribution to the global ECL intensity originates predominantly from the electrode surface. These features govern the remarkable negative optical contrast observed between the mitochondria and the bare electrode surface in SECL. Otherwise, the SECL detection would not be as sensitive as reported in this study. Indeed, SECL microscopy reveals small mitochondria that were hardly detectable by the fluorescence reporters used herein. In addition, in the case of an ECL layer much thicker than the organelles imaged, it would imply that the borders of the mitochondria would be less defined than in fluorescence due to the diffusional broadening and low optical contrast. It did not correspond to the results observed experimentally as illustrated by the intensity profiles extracted from SECL and NADH fluorescence images. Indeed, both profiles were very similar (Figure $2 b$ ). In fact, a close analysis of the luminescence profiles showed even that the full-width at half maximum is smaller for SECL and that the borders are sharper compared to fluorescence. The same behavior is observed when SECL is compared to the MitoTracker fluorescence profile (Figure 2b). This remarkably sharp optical contrast observed in SECL 
supported the fact that the ECL-emitting layer was confined to a micrometric distance from the electrode surface because of the operating "direct oxidation" mechanism.

\section{Conclusion}

In conclusion, we have demonstrated the potentiality of shadow ECL as an alternative surface-confined microscopy to image single subcellular organelles. This label-free approach allowed to visualize functional mitochondria with remarkably sharp negative optical contrast because the electrocatalytic mechanism of the tandem ECL system, $\left[\mathrm{Ru}(\mathrm{bpy})_{3}\right]^{2+} / \mathrm{TPA}$, confined the ECL-emitting region to the electrode surface. The statistical analysis of the colocalization of the shadow ECL spots with the MitoTracker Deep Red dye and the endogenous intramitochondrial NADH biomarker validates the reported methodology for active and intact organelles. In addition, SECL was able to detect small mitochondria, which remain very difficult to observe by fluorescence with the model mitochondrial dyes. The ultrasensitive features of shadow ECL combined with sharp optical contrast contribute to a better understanding of the ECL mechanisms and operating conditions of ECL microscopy in biology. Indeed, this surface-confined approach can also unveil subcellular organelles or non-labeled entities and provides a label-free way to image their morphology. Considering the recent reports on intracellular detection based on various ECL strategies, ${ }^{[16 a, 17 b, 21 b]}$ the ECL imaging of mitochondria in living cells appears possible, even if it is a challenging but rewarding goal. As a versatile electrochemical-based imaging technique, the presented approach opens up manifold experimental opportunities for the development of new ultrasensitive microscopy.

\section{Acknowledgements}

The authors acknowledge the financial support from CNRS, University of Bordeaux, Bordeaux INP and Agence Nationale de la Recherche (MitoCard, ANR-17-CE11-0041; LiCORN, ANR-20-CE29-0006). CC acknowledges the University of Bordeaux for his PhD fellowship (inter-multi-pluri-disciplinary PhD program). Dr Anne Devin and Sylvain Cuvellier (IBGC, CNRS UMR 5095, University of Bordeaux, France) are acknowledged for supplying us with yeast mitochondria and fruitful discussions.

Keywords: electrochemiluminescence $\bullet$ mitochondria $\bullet$ single organelle $\bullet$ microscopy $\bullet$ mechanism $•$ bioelectrochemistry

[1] a) D. G. Nicholls, Methods Mol. Biol. 2018, 1782, 121; b) J. Nunnari, A. Suomalainen, Cell 2012, $148,1145$.

[2] a) M. D. Brand, D. G. Nicholls, Biochem. J. 2011, 435, 297; b) V. S. Vajrala, F. S. Belaidi, G. Lemercier, D. Zigah, M. Rigoulet, A. Devin, N. Sojic, P. Temple-Boyer, J. Launay, S. Arbault, Biosens. Bioelectron. 2019, 126, 672.

[3] a) C. Doerrier, L. F. Garcia-Souza, G. Krumschnabel, Y. Wohlfarter, A. T. Meszaros, E. Gnaiger, Methods Mol. Biol. 2018, 1782, 31; b) M. T. Lin, M. F. Beal, Nature 2006, 443, 787

[4] B. Glancy, Trends. Mol. Med. 2020, 26, 58.

[5] a) E. B. Gokerkucuk, M. Tramier, G. Bertolin, Genes 2020, 11; b) B. Zhu, L. Wu, M. Zhang, Y. Wang, C. Liu, Z. Wang, Q. Duan, P. Jia, Biosens. Bioelectron. 2018, 107, 218; c) P. Gao, W. Pan, N. Li, B. Tang, Chemical Science 2019, 10, 6035; d) X. Luo, X. Gong, L. Su, H. Lin, Z. Yang, X. Yan, J. Gao, Angew. Chem. Int. Ed. 2020, 60, 1403; e) N. Trinh, K. A. Jolliffe, E. J. New, Angew. Chem. Int. Ed. 2020, 59, 20290; f) G. Yang, Z. Liu, R. Zhang, X. Tian, J. Chen, G. Han, B. Liu, X. Han, Y. Fu, Z. Hu, Z. Zhang, Angew. Chem. Int. Ed. 2020, 59, 16154; g) Z. Wu, M. Liu, Z. Liu, Y. Tian, J. Am. Chem. Soc. 2020, 142, 7532.

[6] a) P. B. Allen, B. R. Doepker, D. T. Chiu, Anal. Chem. 2009, 81, 3784; b) G. Quarato, C. Piccoli, R. Scrima, N. Capitanio, Mitochondrion 2011, 11, 764; c) S. Zhang, S. Zhu, L. Yang, Y. Zheng, M. Gao, S. Wang, J.-z. Zeng, X. Yan, Anal. Chem. 2012, 84, 6421; d) A. Schneider, S. Kurz, K. Manske, M. Janas, M. Heikenwaelder, T. Misgeld, M. Aichler, S. FelixWeissmanns, H. Zischka, P. Knolle, D. Wohlleber, Sci. Rep. $2019,9,8492$.

[7] a) S. Hurst, J. Hoek, S.-S. Sheu, J. Bioenerg. Biomembr. 2017, 49, 27; b) L. Boyman, A. K. Coleman, G. Zhao, A. P. Wescott, H. C. Joca, B. M. Greiser, M. Karbowski, C. W. Ward, W. J. Lederer, Arch. Biochem. Biophys. 2019, 666, 31.

[8] a) M. Bonora, P. Pinton, Front. Oncol. 2014, 4, 302; b) S. Javadov, S. Jang, R. Parodi-Rullan, Z. Khuchua, A. V. Kuznetsov, Cell. Mol. Life Sci. 2017, 74,2795

[9] a) K. Hu, T. D. K. Nguyen, S. Rabasco, P. E. Oomen, A. G. Ewing, Anal. Chem. 2021, 93, 41; b) C. Wang, M. Taki, Y. Sato, Y. Tamura, H. Yaginuma Y. Okada, S. Yamaguchi, Proc. Natl. Acad. Sci. 2019, 116, 15817; c) J. Dreier, M. Castello, G. Coceano, R. Cáceres, J. Plastino, G. Vicidomini, I. Testa, Nat. Commun. 2019, 10, 556; d) K. S. Grußmayer, S. Geissbuehler, A. Descloux, T. Lukes, M. Leutenegger, A. Radenovic, T. Lasser, Nat. Commun. 2020, 11, 3023.

[10] a) A. J. Bard, Electrogenerated Chemiluminescence, M. Dekker, New-York, 2004; b) M. Hesari, Z. Ding, J. Electrochem. Soc. 2016,163 , H3116.

[11] a) Z. Liu, W. Qi, G. Xu, Chem. Soc. Rev. 2015, 44, 3117; b) N. Sojic, Analytical Electrogenerated Chemiluminescence: From Fundamentals to Bioassays, Royal Society of Chemistry (RSC) Publishing, 2020.

[12] J. Zhang, S. Arbault, N. Sojic, D. Jiang, Annu. Rev. Anal. Chem. 2019, 12, 275

[13] C. Ma, Y. Cao, X. Gou, J.-J. Zhu, Anal. Chem. 2020, 92, 431.

[14] a) Y. Chen, D. Zhao, J. Fu, X. Gou, D. Jiang, H. Dong, J.-J. Zhu, Anal. Chem. 2019, 91, 6829; b) L. Xu, Y. Li, S. Wu, X. Liu, B. Su, Angew. Chem. Int. Ed. 2012, 51, 8068; c) M.-J. Zhu, J.-B. Pan, Z.-Q. Wu, X.-Y. Gao, W. Zhao, X.-H. Xia, J.-J. Xu, H.-Y. Chen, Angew. Chem. Int. Ed 2018, 57, 4010; d) C. Ma, H.-F. Wei, M.-X. Wang, S. Wu, Y.-C. Chang, J. Zhang, L.-P. Jiang, W. Zhu, Z. Chen, Y. Lin, Nano Lett. 2020, 20, 5008; e) A. J. Wilson, K. Marchuk, K. A. Willets, Nano Lett. 2015, 15, 6110; f) M. W. Glasscott, J. E. Dick, J. Phys. Chem. Lett. 2020, 11, 4803; g) J. E. Dick, C. Renault, B.-K Kim, A. J. Bard, Angew. Chem. Int. Ed. 2014, 53, 11859; h) C. Ma, W. Wu, L. Li, S. Wu, J. Zhang, Z. Chen, J.-J. Zhu, Chem. Sci. 2018, 9, 6167; i) A. Fiorani, D. Han, D. Jiang, D. Fang, F. Paolucci, N. Sojic, G. Valenti, Chem. Sci. 2020, 11, 10496; j) P. Dutta, D. Han, B. Goudeau, D. Jiang, D. Fang, N. Sojic, Biosens. Bioelectron. 2020, 165, 112372; k) A. Zanut, F. Palomba, M. Rossi Scota, S. Rebeccani, M. Marcaccio, D. Genovese, E. Rampazzo, G. Valenti, F. Paolucci, L. Prodi, Angew. Chem. Int. Ed. 2020, 59, 21858; I) A. Zanut, A. Fiorani, S. Canola, T. Saito, N. Ziebart, S. Rapino, S. Rebeccani, A. Barbon, T. Irie, H.-P. Josel, F. Negri, M. Marcaccio, M. Windfuhr, K. Imai, G. Valenti, F. Paolucci, Nat. Commun. 2020, 11, 2668; m) W. Guo, H. Ding, P. Zhou, Y. Wang, B. Su, Angew. Chem. Int. Ed. 2020, 59, 6745; n) M.-M. Chen, W. Zhao, M.-J. Zhu, X.-L. Li, C.-H. Xu, H.-Y. Chen, J.-J. Xu, Chem. Sci. 2019, 10, 4141.

[15] H. Ding, W. Guo, P. Zhou, B. Su, Chem. Commun. 2020, 56, 8249. 
[16] a) Y. Wang, R. Jin, N. Sojic, D. Jiang, H.-Y. Chen, Angew. Chem. Int. Ed. 2020, 59, 10416; b) H. Zhang, W. Gao, Y. Liu, Y. Sun, Y. Jiang, S. Zhang, Anal. Chem. 2019, 91, 12581; c) G. Ma, J. Zhou, C. Tian, D. Jiang, D. Fang, H. Chen, Anal. Chem. 2013, 85, 3912; d) J. Zhou, G. Ma, Y. Chen, D. Fang, D. Jiang, H.-y. Chen, Anal. Chem. 2015, 87, 8138.

[17] a) G. Valenti, S. Scarabino, B. Goudeau, A. Lesch, M. Jović, E. Villani, M. Sentic, S. Rapino, S. Arbault, F. Paolucci, N. Sojic, J. Am. Chem. Soc. 2017, 139, 16830; b) S. Voci, B. Goudeau, G. Valenti, A. Lesch, M. Jović, S. Rapino, F. Paolucci, S. Arbault, N. Sojic, J. Am. Chem. Soc. 2018, $140,14753$.

[18] N. Wang, H. Gao, Y. Li, G. Li, W. Chen, Z. Jin, J. Lei, Q. Wei, H. Ju, Angew. Chem. Int. Ed. 2021, 60.

[19] a) H. Ding, W. Guo, B. Su, Angew. Chem. Int. Ed. 2020, 59, 449; b) H. Gao, W. Han, H. Qi, Q. Gao, C. Zhang, Anal. Chem. 2020, 92, 8278; c) J. Zhang, R. Jin, D. Jiang, H.-Y. Chen, J. Am. Chem. Soc. 2019, 141, 10294.

[20] H. Ding, P. Zhou, W. Fu, L. Ding, W. Guo, B. Su, Angew. Chem. Int. Ed. 2021, DOI: 10.1002/anie.202101467.

[21] a) H. Qi, C. Zhang, Anal. Chem. 2020, 92, 524; b) C. Ma, S. Wu, Y. Zhou, H.-F. Wei, J. Zhang, Z. Chen, J.-J. Zhu, Y. Lin, W. Zhu, Angew. Chem. Int. Ed. 2021, 60, 4907; c) N. Sojic, D. Han, B. Goudeau, D. Manojlovic, D. Jiang, D. Fang, Angew. Chem. Int. Ed. 2021, $60,7686$.

[22] a) L. Scorrano, V. Petronilli, R. Colonna, F. Di Lisa, P. Bernardi, J. Biol. Chem. 1999, 274, 24657; b) J. R. Gledhill, J. E. Walker, Biochem J. 2005, 386, 591.

[23] a) D. Lasne, G. A. Blab, F. De Giorgi, F. Ichas, B. Lounis, L. Cognet, Opt. Express 2007, 15, 14184; b) S. Adhikari, P. Spaeth, A. Kar, M. D. Baaske, S. Khatua, M. Orrit, ACS Nano 2020.

[24] a) S.-Y. Choi, P. Huang, G. M. Jenkins, D. C. Chan, J. Schiller, M. A. Frohman, Nat. Cell Biol. 2006, 8, 1255; b) R. Zhou, A. S. Yazdi, P. Menu, J. Tschopp, Nature 2011, 469, 221.

[25] E. Suraniti, V. S. Vajrala, B. Goudeau, S. P. Bottari, M. Rigoulet, A. Devin, N. Sojic, S. Arbault, Anal. Chem. 2013, $85,5146$.

[26] W. Miao, J.-P. Choi, A. J. Bard, J. Am. Chem. Soc. 2002, 124, 14478.

[27] M. Sentic, M. Milutinovic, F. Kanoufi, D. Manojlovic, S. Arbault, N. Sojic, Chem. Sci. 2014, 5, 2568

[28] B. Liu, S. A. Rotenberg, M. V. Mirkin, Proc. Natl. Acad. Sci. USA 2000, 97, 9855.

[29] S. Todisco, G. Agrimi, A. Castegna, F. Palmieri, J. Biol. Chem. 2006, 281, 1524.

[30] a) V. S. Vajrala, E. Suraniti, M. Rigoulet, A. Devin, N. Sojic, S. Arbault, Integr. Biol. 2016, 8, 836; b) S. M. Rafelski, M. P. Viana, Y. Zhang, Y.-H. M. Chan, K. S. Thorn, P. Yam, J. C. Fung, H. Li, L. d. F. Costa, W. F. Marshall, Science 2012, 338, 822; c) S. van de Linde, M. Sauer, M. Heilemann, J. Struct. Biol. 2008, 164, 250.

[31] a) W. Wang, S. Wang, Q. Liu, J. Wu, N. Tao, Langmuir 2012, 28, 13373; b) E. Kreysing, H. Hassani, N. Hampe, A. Offenhäusser, ACS Nano 2018, 12,8934 .

[32] a) O. V. Klymenko, I. Svir, C. Amatore, ChemPhysChem 2013, 14, 2237; b) Z. Chen, Y. Zu, J. Phys. Chem. C 2008, 112, 16663; c) Z. Chen, Y. Zu, J. Phys. Chem. C 2009, 113, 21877; d) E. Daviddi, A. Oleinick, I. Svir, G. Valenti, F. Paolucci, C. Amatore, ChemElectroChem 2017, 4, 1719.

[33] G. Valenti, A. Fiorani, H. Li, N. Sojic, F. Paolucci, ChemElectroChem 2016, 3, 1990.

[34] a) W. Guo, H. Ding, C. Gu, Y. Liu, X. Jiang, B. Su, Y. Shao, J. Am. Chem. Soc. 2018, 140, 15904; b) R. Qiu, X. Zhang, H. Luo, Y. Shao, Chem. Sci. 2016, 7, 6684; c) J. Hu, N. Zhang, P.-K. Zhang, Y. Chen, X.-H. Xia, H.-Y. Chen, J.-J. Xu, Angew. Chem. Int. Ed. 2020, 59, 18244; d) P. Pastore, D. Badocco, F. Zanon, Electrochim. Acta 2006, 51, 5394.

[35] Y. Zu, A. J. Bard, Anal. Chem. 2000, 72, 3223.

[36] F. Kanoufi, Y. Zu, A. J. Bard, J. Phys. Chem. B 2001, 105, 210.

[37] W. Guo, P. Zhou, L. Sun, H. Ding, B. Su, Angew. Chem. Int. Ed. 2021, 60, 2089.

[38] Y. Wang, W. Guo, Q. Yang, B. Su, J. Am. Chem. Soc. 2020, 142, 1222. 